INFLAMMATION AND INFLAMMATORY BOWEL DISEASE

\title{
No seasonality in month of birth of inflammatory bowel disease cases: a prospective population based study of British under 20 year olds
}

\author{
T R Card, A Sawczenko, B K Sandhu, R F A Logan
}

Gut 2002;51:814-815

$P$ renatal or perinatal environmental exposures have been postulated to change the risk of inflammatory bowel disease (IBD) later in life. ${ }^{1}$ One manifestation of this, perhaps because of early and seasonal infectious exposure, might be an association between the month of birth and risk of developing IBD later in life. ${ }^{2-4}$ Two recent publications, one from the UK, ${ }^{3}$ and the other from Denmark ${ }^{4}$ have examined cohorts of Crohn's patients for such seasonality of birth. The findings of these two studies as well as their interpretations differ markedly. The British study was of adult patients diagnosed between 1972 and 1989 by four regional IBD registers in which a weak association was found between Crohn's disease (CD) and birth in the first half of the year. The second (Danish) study examined national computerised hospital discharge records of children and young people aged less than 20 years coded between 1977 and 1992 for CD. This second study found an apparently stronger association with month of birth with the peak risk being for those born during August with a "sine wave" variation in risk between those born in different months of the year. The authors suggest that the differences compared with the British study might be explained by differences in age mix and nationality of the subjects, and further suggested that their findings give support to the importance of in utero and early childhood events in the aetiology of CD.

In view of these inconsistent findings, and the possibility that they may in part be explained by the differences in analytical technique as well as the age mix of the cases, we undertook an analysis of the national cohort of British IBD patients aged less than 20 years collected prospectively by the British Paediatric Surveillance Unit (BPSU) and the British Society of Gastroenterology Research Unit (BSGRU). We analysed this series using similar methods to those used by both the previous studies and in addition extended the statistical analysis to take into account possible shortcomings in them.

\section{METHODS AND RESULTS}

Patients aged 0-16 years collected by the BPSU-BSGRU survey in the British Isles have previously been described ${ }^{5}$ and to the published data were added notifications of patients aged 16-20 years from the BSGRU survey. In total there were 1040 incident cases of IBD diagnosed in those aged 0-20 years in the UK during the study period of June 1998 to June 1999. The median year of birth of these cases was 1984 with a mode in 1983 and a standard deviation of 3.6 years. There were 634 cases of CD, 277 cases of ulcerative colitis (UC), 12 cases of orofacial granulomatosis, and 117 cases of indeterminate colitis.

The monthly numbers of live births during the period when children were born (1978-1998) were obtained for England, Scotland, Wales, and Northern Ireland (table 1). The expected number of cases for each month were obtained by dividing the observed cases born in each year between the year's months in the ratio of the total births within the months of that year. The monthly expected cases were then summed for each month over all 20 years (table 1). The "rate" for this incident cohort was calculated as the total cases born in a particular month divided by the total births over the 20 years in that month.

The numbers of cases expected were compared with the observed data using a $\chi^{2}$ test. There was no statistically significant difference between the observed and expected numbers for IBD $(p=0.88), C D(p=0.17)$, or UC $(p=0.77)$. These differences remained non-significant when the data were analysed by half year (January to June and July to December) compared with month ( $p=0.40$ for IBD, $p=0.50$ for $\mathrm{CD}$, and $\mathrm{p}=0.70$ for $\mathrm{UC}$ ).

Periodic regression models similar to those previously described in the Danish study, ${ }^{4}$ (that is, linear regression ${ }^{6}$ models incorporating as covariates in a linear regression, terms giving the value of sine $(\mathrm{i} \times \mathrm{month} \times 30 \times \pi / 180)$ and similar cosine terms) with one sine and one cosine variable were then used to model the relation between "rate" and month, and also between observed and expected numbers of cases by month. These models were then compared with similar models excluding the periodic terms - that is, those with constant "rate" throughout the year. Figure l shows that these models including the periodic terms provided a better fit with the data than the models without them. However, a comparison between the models using a likelihood ratio test showed that the improvement in fit as a result of the inclusion of periodic terms was not significant ( $p=0.84$ for all IBD, $p=0.87$ for $\mathrm{CD}$, and $\mathrm{p}=0.18$ for $\mathrm{UC}$ ). Similarly comparing models examining the relation between observed and expected numbers, the likelihood ratio test showed that models incorporating periodic terms did not fit significantly better than those without ( $\mathrm{p}=0.95$ for all IBD, $\mathrm{p}=0.90$ for $\mathrm{CD}$, and $\mathrm{p}=0.42$ for $\mathrm{UC}$ ).

As a single sine cosine pair constrains the model to have only one maximum and one minimum within the year, models were refitted with two and then three sine cosine pairs to permit a closer fit between model and data (for the first pair $i=1$, the second $i=2$, etc). Again the likelihood ratio tests showed no significant improvement in fit from using periodic terms in any of these models.

\section{DISCUSSION}

This study found no significant relation between month of birth and the risk of developing inflammatory bowel diseases in the early years of life; findings consistent with the previous British study of older patients with CD. As the current cohort includes cases in the same age range as the recent Danish study, ${ }^{4}$ differences in age structure cannot explain the difference between these and the Danish findings. Differences

Abbreviations: IBD, inflammatory bowel disease; $C D, C$ rohn's disease; UC, ulcerative colitis 
Table 1 Birth data and observed/expected cases of inflammatory bowel disease in the United Kingdom

\begin{tabular}{|c|c|c|c|c|c|c|c|c|c|c|c|c|}
\hline & Jan & Feb & Mar & Apr & May & June & July & Aug & Sept & Oct & Nov & Dec \\
\hline UK births 1978-98 (thousands) & 1302 & 1202 & 1336 & 1296 & 1355 & 1327 & 1377 & 1344 & 1339 & 1330 & 1245 & 1257 \\
\hline$C D$ cases observed & 69 & 45 & 44 & 59 & 49 & 58 & 59 & 56 & 61 & 43 & 52 & 39 \\
\hline$C D$ cases expected & 52 & 48 & 54 & 52 & 55 & 53 & 55 & 54 & 54 & 54 & 50 & 50 \\
\hline "Rate" of CD per 100000 births & 5.3 & 3.7 & 3.3 & 4.6 & 3.6 & 4.4 & 4.3 & 4.2 & 4.6 & 3.2 & 4.2 & 3.1 \\
\hline UC cases observed & 26 & 26 & 23 & 17 & 29 & 20 & 20 & 25 & 20 & 24 & 20 & 27 \\
\hline UC cases expected & 23 & 21 & 24 & 23 & 24 & 23 & 24 & 24 & 24 & 23 & 22 & 22 \\
\hline "Rate" of UC per 100000 births & 2.0 & 2.2 & 1.7 & 1.3 & 2.1 & 1.5 & 1.5 & 1.9 & 1.5 & 1.8 & 1.6 & 2.2 \\
\hline IBD cases observed & 103 & 81 & 80 & 88 & 88 & 91 & 88 & 92 & 89 & 80 & 82 & 78 \\
\hline IBD cases expected & 86 & 79 & 87 & 86 & 90 & 87 & 91 & 89 & 89 & 88 & 82 & 83 \\
\hline "Rate" of all IBD per 100000 births & 7.9 & 6.7 & 6.0 & 6.8 & 6.5 & 6.9 & 6.4 & 6.8 & 6.6 & 6.0 & 6.6 & 6.2 \\
\hline
\end{tabular}

$C D$, Crohn's disease; UC, ulcerative colitis; IBD, all cases of inflammatory bowel disease. Expected, the number of cases that would have been born in the calendar month if all differences in the number of cases per month were attributable to variation in the total number of births. "Rates", calculated from the observed cases and total births. See text for definition of "rate".

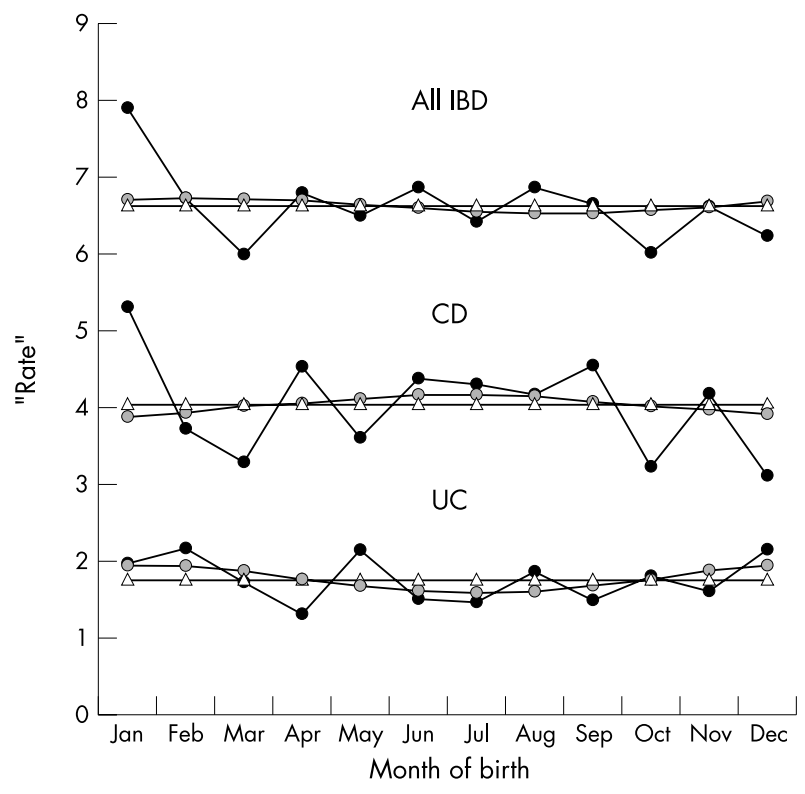

Figure 1 Observed and modelled "rates" of inflammatory bowel disease. Observed rates (dark lines), expected from periodic modelling (grey lines), expected from non-periodic modelling (triangles). See text for explanation of "rate".

between the two studies include methodology of case ascertainment, time period of case diagnosis, and statistical technique. Although we have used statistical techniques similar to those previously described one important difference is that we have quoted a significance test for the periodic regression models.
Although it is possible that IBD may in part result from the effect of exposures early in life, we have found no evidence of seasonality of birth month. We do not believe that this study or the previous analyses give good evidence to suggest that important aetiological factors acting specifically at or around the time of birth are likely to be found among exposures varying by season, and despite their shortcomings believe that it is unlikely that any substantial seasonal variation has been overlooked.

\section{Authors' affiliations}

T R Card, R F A Logan, Division of Public Health Sciences, Nottingham, UK

A Sawczenko, B K Sandhu, Department of Gastroenterology, The Children's Hospital, Bristol, UK

Correspondence to: Professor R F A Logan, Division of Public Health Sciences, University Hospital, Nottingham, UK

richard.logan@nottigham.ac.uk

Accepted for publication 30 April 2002

\section{REFERENCES}

1 Ekbom A, Zack M, Adami HO, et al. Is there clustering of inflammatory bowel disease at birth? Am J Epidemiol 1991;134:876-86.

2 Ekbom A, Helmick C, Zack M, et al. The epidemiology of inflammatory bowel disease: a large, population-based study in Sweden. Gastroenterology 1991;100:350-8.

3 Haslam N, Mayberry JF, Hawthorne AB, et al. Measles, month of birth, and Crohn's disease. Gut 2000;47:801-3.

4 Sorensen HT, Pedersen L, Norgard B, et al. Does month of birth affect risk of Crohn's disease in childhood and adolescence? BM 2001;323:907

5 Sawczenko A, Sandhu BK, Logan RF, et al. Prospective survey of childhood inflammatory bowel disease in the British Isles. Lancet 2001;357: 1093-4.

6 Armitage P, Berry G. Statistical methods in medical research. 3rd edn. Oxford: Blackwell Scientific, 1994:312-48. 